\title{
An Interesting Case of a Large Cystic Mesothelioma of the Omentum in a Pediatric Patient
}

\author{
Gnanasekar Periyasamy ${ }^{1}$ Sivasundhar Kumarasamy ${ }^{1} \quad$ Karumuri Srinivas Sekhar ${ }^{1}$ \\ Malathi Vaithyanathan ${ }^{1}$ Saravanakumar Sengottaiyan ${ }^{1}$ Saravanan Thangam Shanmugasundaram ${ }^{1}$ \\ Kumaragurubaran Gnanasambandam²
}

1Department of Radiology, Billroth Hospital, Chennai, Tamil Nadu, India

${ }^{2}$ Department of Surgery, Billroth Hospital, Chennai, Tamil Nadu, India

\begin{abstract}
Address for correspondence Gnanasekar Periyasamy, MBBS, DMRD, DNB, Department of Radiology, Billroth Hospital, 43, Lakshmi Talkies Road, Shenoy Nagar, Chennai 600030, Tamil Nadu, India (e-mail: drgnanasekarmbbs1989@gmail.com).
\end{abstract}

J Gastrointestinal Abdominal Radiol ISGAR 2019;2:74-76

\begin{abstract}
We present an interesting case of a large intra-abdominal cystic lesion in a 14-year-old girl who presented with abdominal discomfort and pain. The patient was evaluated

Keywords

- omental cyst

- mesenteric cyst

- mesothelioma with magnetic resonance imaging (MRI) and found to have a large septated cystic lesion arising from the gastrohepatic omentum. She underwent surgery, and the diagnosis of cystic mesothelioma was confirmed in the histopathology. To the best of our knowledge, only a few cases of benign omental mesothelial cysts have been reported.
\end{abstract}

\section{Case Presentation}

A 14-year-old girl presented to outpatient department (OPD) with abdominal discomfort, pain, and occasional vomiting for past 6 months. On clinical examination, the clinician suspected an intra-abdominal mass, and the patient was referred for magnetic resonance imaging (MRI). Routine investigations were within normal limits.

MRI (GE 1.5T, Signa HDxt, Milkaukee, Wisconsin, USA) abdomen with and without contrast was performed. Axial and coronal T2 SSFSE with and without fat saturation, T1 LAVA, precontrast T1 FSE axial fat saturation, diffusion, 3D MRCP with respiratory gating and breath holding sequences were obtained. Following contrast administration $(6 \mathrm{ml}$ of Gadobenate dimeglumine, Milano, Italy), axial and coronal T1 FSE fat saturated sequences were obtained.

Alarge 17-×15.7-×6-cm thin-walled, well-encapsulated, and multilobulated T2W hyperintense cystic lesion, with few thin internal septations in the supramesocolic compartment extending into the omentum ( - Fig. 1). There was no restriction of diffusion. The lesion was seen involving the gastrohepatic space and lesser sac closely abutting the adjoining left lobe of the liver, stomach, and pancreas (-Fig. 2). The cyst extends to the right side along the omentum into the right hypochondrium and lumbar region and inferiorly up to the right iliac fossa region. The ascending colon was displaced posteriorly and the adjoining small bowel loops postero medially.

\section{received}

January 1, 2019

accepted after revision

February 7, 2019

Following intravenous contrast administration, there is no demonstrable enhancement of the cyst wall with mild enhancement of the septae. No enhancing solid component/ mural nodularity was noted within the cyst ( - Fig. 3). Diagnosis of a large benign mesothelial omental cyst was made.

The patient then underwent surgery for removal of the omental cyst. Intraoperatively, the cyst was found to be arising from the gastrohepatic omentum. The cyst wall was found to be translucent and thin ( $\boldsymbol{- F i g .} \mathbf{4}$ ). The cyst was excised in toto and sent for histopathology.
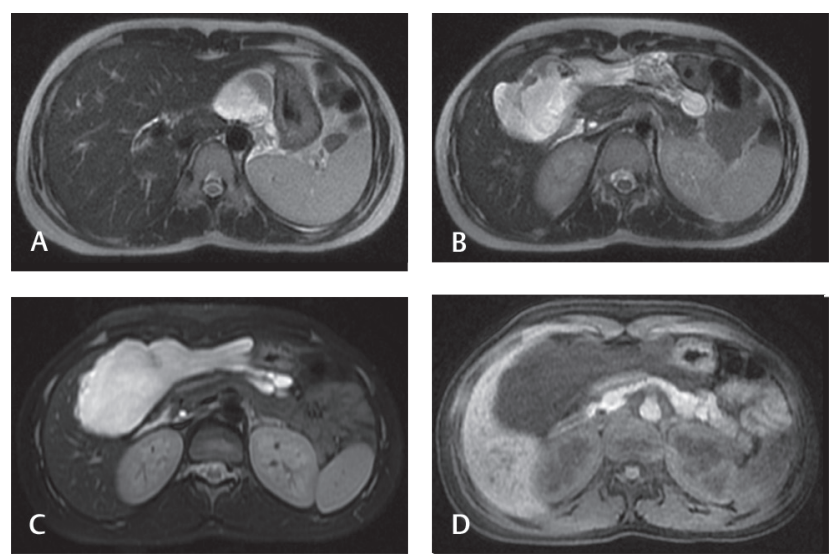

Fig. 1 (A, B) Axial T2 weighted, (C) axial T2 fat sat, and (D) axial T1 LAVA images showing a large septated cystic lesion arising from the lesser omentum abutting the left lobe of liver and lesser curvature of stomach. No solid areas noted in the cyst. 

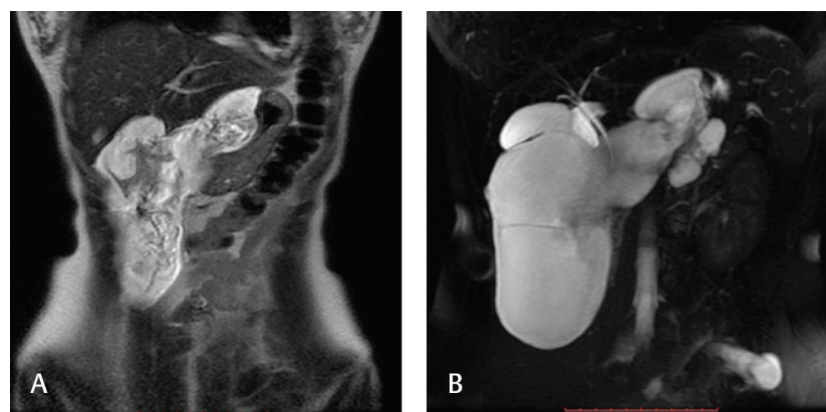

Fig. 2 (A) Coronal T2W and (B) coronal MRCP images showing a large septated cystic lesion arising from the lesser omentum and extending into the right lumbar and iliac region.
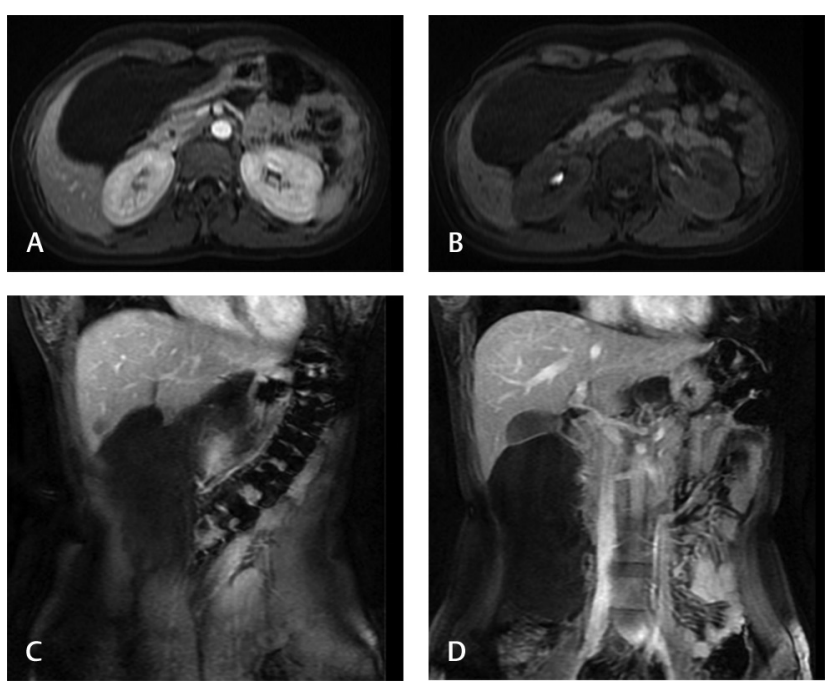

Fig. 3 (A, B) Postcontrast axial T1W fat sat and (C, D) postcontrast coronal T1W fat sat images showing no significant enhancement of the cyst wall and no enhancing solid areas.

The histopathology report showed multicystic lesion with fibroadipose wall lined focally by flattened cuboidal cells. The stroma showed few small mesothelial clusters. Cholesterol clefts with giant cells and scattered lymphocytic infiltration were noted. There was no evidence of malignancy ( - Fig. 5).

\section{Discussion}

Mesenteric and omental cysts are uncommon intraabdominal masses, generally of a benign nature. The incidence of mesenteric cysts is nearly 1 of 105,000 hospital admissions, and the incidence of omental cysts is even rarer. ${ }^{1}$ These cysts are more common in adults, with only $25 \%$ of cases diagnosed before the age of 10 years. $^{2}$ Various etiologies have been proposed for the omental and mesenteric cysts including benign proliferation of the ectopic mesenteric lymphatics that do not communicate with the rest of the lymphatic system, failure to join the embryonic lymphatic spaces to the venous system, failure of the fusion of mesenteric leaves, deficiency of the normal

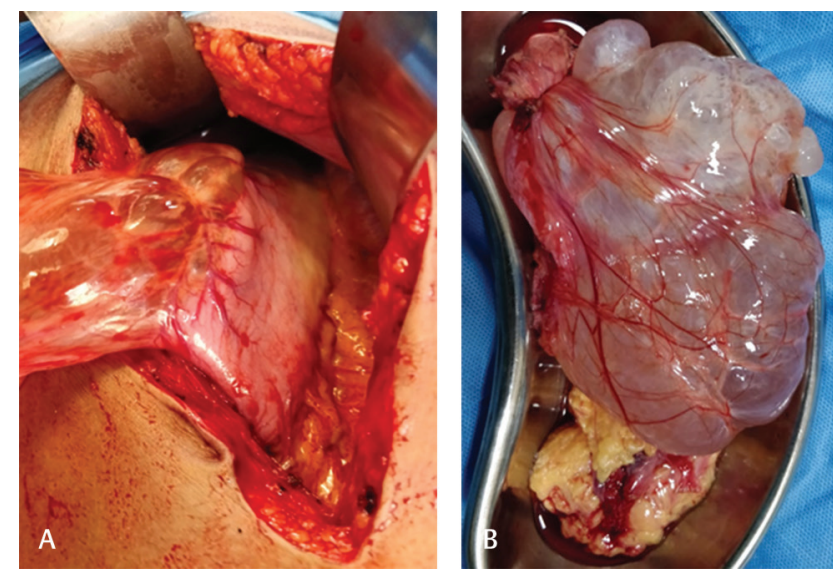

Fig. 4 (A) Intraoperative image showing the cystic lesion attached to lesser omentum and (B) gross specimen of the cystic lesion.

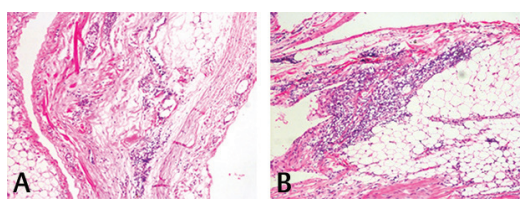

Fig. 5 (A, B) Histopathology sections showing multicystic lesion with fibroadipose wall lined focally by flattened cuboidal cells. The stroma shows few small mesothelial clusters. Cholesterol clefts with giant cells and scattered lymphocytic infiltration are noted. There is no evidence of malignancy.

lymphaticovenous shunts, trauma, neoplasm, and localized degeneration of lymph nodes. ${ }^{3}$

These cysts are present either incidentally, with nonspecific abdominal complaints or with acute abdominal pain. The spectrum of presentation depends primarily on the location and the size of the cyst. Any emerging complications, including enlargement, intracystic hemorrhage, torsion, infection, ${ }^{4}$ or rupture are common indications of a surgical excision. Ultrasound is a very sensitive and specific radiologic imaging modality used not only for the diagnosis but also for the follow-up of these cysts, even in the prenatal period. ${ }^{5}$ Despite providing additional information about the location, size, and invasion of the cysts into the surrounding tissues, computed tomography is not generally necessary while evaluating mesenteric cysts in childhood because of the high radiation exposure.

MRI could be an alternative for evaluation, especially in prenatal cystic lesions. The treatment includes the complete excision of the cystic mass by laparotomy. Minimally invasive surgery (MIS) is a surgical treatment option. These cysts can be excised either laparoscopically or in a laparoscopy-assisted manner. ${ }^{6-8}$ Sclerosing agent therapies either alone or followed by marsupialization can be used with a moderate risk of recurrence in the case of complete excision failure.

\section{Conflict of Interest}

None declared. 
76 Large Cystic Mesothelioma of the Omentum Periyasamy et al.

\section{References}

1 Ricketts RR. Mesenteric and omental cysts. In: Grosfeld JL, O'Neill JAJ, Coran AG, Fonkalsrud EW, eds. Pediatric Surgery. Philadelphia, PA: Mosby Elsevier; 2006:1399-1406

2 Pampal A, Yagmurlu A. Successful laparoscopic removal of mesenteric and omental cysts in toddlers: 3 cases with a literature review. J Pediatr Surg 2012;47(8):e5-e8

3 Lin JI, Fisher J, Caty MG. Newborn intraabdominal cystic lymphatic malformations. Semin Pediatr Surg 2000;9(3):141-145

4 Kim E-J, Lee S-H, Ahn BK, Baek SU. Acute abdomen caused by an infected mesenteric cyst in the ascending colon: a case report. J Korean Soc Coloproctol 2011;27(3):153-156
5 Konen O, Rathaus V, Dlugy E, et al. Childhood abdominal cystic lymphangioma. Pediatr Radiol 2002;32(2):88-94

6 Esposito C, Alicchio F, Savanelli A, Ascione G, Settimi A. One-trocar ileo-colic resection in a newborn infant with a cystic lymphangioma of the small-bowel mesentery. J Laparoendosc Adv Surg Tech A 2009;19(3):447-449

7 Kuga T, Inoue T, Taniguchi S, Zempo N, Esato K. Laparoscopic surgery in infants with intra-abdominal cysts: two case reports. JSLS 2000;4(3):243-246

8 Al-Zaiem MM. Assisted laparoscopic excision of huge abdominal cysts in newborns and infants using the umbilical laparoscopic port incision. J Pediatr Surg 2011;46(7): 1459-1463 